\title{
Analysis of Variation in Relative Mobility with Traffic Load in Wireless Mobile Network
}

\author{
Gaurav Sharma ${ }^{1}$ and Manjeet Kharub $^{2}$ \\ ${ }^{1}$ Asst. Professor, CVR College of Engineering/ECE Department, Hyderabad, India \\ Email: ergaurav209@yahoo.co.in \\ ${ }^{2}$ Asst. Professor, CVR College of Engineering/ Mechanical Engineering Department, Hyderabad, India \\ Email: manjeetkharub@gmail.com
}

\begin{abstract}
Call blocking and calls dropping in cellular communication system are the critical issues. Sometimes it occurs due to less network coverage, and handoff mechanism. Handoff is the process of moving from one cell to another cell, when the call is in the progress. This paper presents one of the handoff mechanism using different pathloss models to avoid the call blocking and call dropping probability using Gaussian noise and hysteresis margin. Due to small size of the cell, more handovers occur. Since, capacity of the network increases due to small size of the cell. It also increases the delay and switching load in the network. Quality of Service (QoS) also depends on the quick handoff mechanism, as unnecessary handoffs affect the QoS and should be avoided. In this paper, the proposed scheme supports better QoS and grade of Service (GoS).
\end{abstract}

Index Terms: Cellular network, Heterogeneous Network, Handoff, QoS, GoS.

\section{INTRODUCTION}

In wireless mobile communication, coverage and connectivity are the main issues that should be handled by the service provider, due to poor coverage and connectivity, the QoS degrades [1]. The user needs full service irrespective of any area. In cellular communication system, limited bandwidth is provided to the service providers. It is necessary for the service providers to manage all the communication within this limited bandwidth [2, 3]. Therefore, the frequency reuse concept is being used to make it possible. To achieve this, the coverage region is distributed into the smaller area called cells. By using the frequency reuse concept, the capacity is also increased. More number of users can avail the service; hence coverage and connectivity are increased. But due to increased capacity, interference may be increased $[4,5]$.

In each smaller region, transmitter and receiver are used to serve the users within its coverage. Transmitter and receiver of each cell are connected through wireless link. To provide the constant service, ongoing calls can be transferred from the presently serving cell to different cell when the users move in between the cells [1, 3, 6]. Heavy traffic flow in the network is generated due to large number of users in the network. When such a large number of users are present simultaneously in the particular cell, then that cell becomes hot spot cell. Therefore, it is necessary to design such a mechanism, which can handle the traffic routing properly, and allow the users to automatically move from the congested cell to make the network balance [2-4]. Network balancing [7] is the ability to balance the traffic between two links without using complex routing protocols. This capability balances network sessions like Web, email, etc. over multiple connections in order to spread out the amount of bandwidth used by each user, thus increasing the total amount of bandwidth available [5]. In this paper, analysis of variation in relative mobility with traffic load in heterogeneous mobile network has been carried out. To balance the traffic load in the hotspot cell, an algorithm is proposed in this paper.

\section{HeTERogenous NeTWORK AND HANDOFF MECHANISM}

In cellular system, mobile station and base station are the basic units. These both units have different processing power, memory, communication range, power, etc. which make the system Heterogeneous Network commonly called as HetNets. Several mobile users are served by the single base station, due to which base station is having some high processing capabilities. On the other hand, homogeneous networks are the networks in which processing power, memory, communication range, etc. are kept same. But it is not usually considered in cellular systems $[8,9,10]$. As the traffic demand grows and the number of RF chains change, the network relies on cell splitting or additional carriers to overcome capacity and link budget limitations and maintain uniform user experience. This deployment process is complex and iterative [7]. A more flexible deployment model is needed for operators to improve broadband user experience in a ubiquitous and cost-effective way. Continuous service from the base station depends on the location of the mobile user and its mobility i.e. whether it is in the coverage range of the base station or not. If it moves out from the coverage range of the present serving cell, then it is not guaranteed to provide continuous service. Operations in HetNets are somewhat critical due to managing different parameters.

However portable administrators are hoping to embrace the heterogeneous cell arrangement, HetNet is the way to deal with, empower them to attain their objectives for coverage and capacity $[11,12]$.

A handoff alludes to the way toward exchanging an ongoing call or information session from one serving cell in a cellular network to different or starting with one direct in a phone then onto the next. An all-Around actualized handoff is essential for conveying continuous support of a guest or information session client. In Europe and different nations, a 
handoff is known as a handover. Cell systems are made out of cells, each of which is equipped for giving broadcast communications administration to supporters meandering inside them $[2,7]$. Every cell can just serve up to a specific region and number of endorsers. Consequently, when any of these two cutoff points is achieved, a handoff follows.

For example, if an endorser takes off the service from present serving cell or moves out of the coverage zone of a specific cell while entering another, a handoff happens between the two cells [4]. The duties of the cell that served the call previous to the handoff is exchanged to the second cell $[12,13]$. A handoff may likewise be activated when the number of subscribers utilizing a specific cell has just achieved the cell's most extreme breaking point (capacity). Each time a mobile user goes starting with one cell then onto the next, the system naturally switches the obligation of coverage from one serving cell to other. It is called, "Hand Off" mechanism [8].

With the appearance of CDMA (Code Division Multiple Access) frameworks where same kind of networks can be utilized by a few mobiles and where it is conceivable to nearby base stations or cell segments to utilize a similar recurrence channel there are various diverse sorts of handoff that can be executed. For the most part, handoff in versatile systems can be arranged into two kinds [1]:

\section{- Hard handoff}

\section{- Soft handoff}

Although these types of handovers empower the mobile users to be associated with an alternate base station or distinctive cell part, they are accomplished in marginally extraordinary ways and are accessible under various situations.

At a time, two connections can be maintained; such type of technology is performed in $3 \mathrm{G}$ CDMA, where neighbouring cell provides the connection to the new incoming mobile users before their connection break from the old base station. Since, the neighbouring cell also works on similar frequency, due to which soft handoff is possible. In this type of channel accessing technique, to avoid the interference from the neighbouring cells, unique codes are used for each mobile user [7].

\section{CDMA System ANd Handoff Measurement PROCEDURE}

CDMA (Code-Division Multiple Access) is the multiplexing technique that is used to access the channel. In CDMA, a large frequency set is used by the wireless channel to access it by using a code of $n$ bits, where $n$ is large number greater than 50 . The unique codes consist of binary digits such as $1 \mathrm{~s}$ and $0 \mathrm{~s}$. In CDMA system, ultrahigh-frequency (UHF) of frequency range between 800 $\mathrm{MHz}$ to $1.9 \mathrm{GHz}$ is used by the users to make the accessible speed faster and transmission without interference [1, 3]. In this interference is very less from the neighbouring cells due to use of the unique code by the mobile users.

The CDMA system mainly uses direct sequence spread spectrum and efficient error control codes. In this technique, all the information is transmitted at same frequency at same time with different codes, due to which inter cell and intra cell interference becomes very less. By using physical layer schemes, it is possible to use the frequency again in the cells unlike the other multiple access techniques. To minimize the inter cell interference, fast power control mechanism is used. The forward channel in CDMA is between the base station and mobile station. In this forward channel, QPSK (Quadrature Phase Shift Keying) modulation is used. In CDMA, the air interface is by far the most complex of all multiple access techniques [11, 12]. This technique uses spreading code methodology to spread the symbols before sending these codes from transmitter. On the receiver side, these codes are de-spread using correlator to generate the original message signal. However, CDMA uses the unique codes for the information transmission; due to it in CDMA soft handoff is employed.

The handoff system can be divided into three stages: measurement, decision and execution phases as shown in Fig. 1. In the measurement phase, measurement of the information required for handoff is carried out. Typically, it is measured by the mobile station by calculating the $E_{c} / I_{0}$ of the Common Pilot Channel (CPICH) of its present serving cell and alternate neighbouring cells. In the second phase of handoff procedure, first step results (measurement results) are compared with the predefined threshold values, that is decided by the mobile whether the handoff should be initiated or not. In the execution phase, based on the decision performed by decision phase, handoff process is completed. As soon as the process is completed, different parameters are changed according to the handoff type i.e. hard handoff or soft handoff.

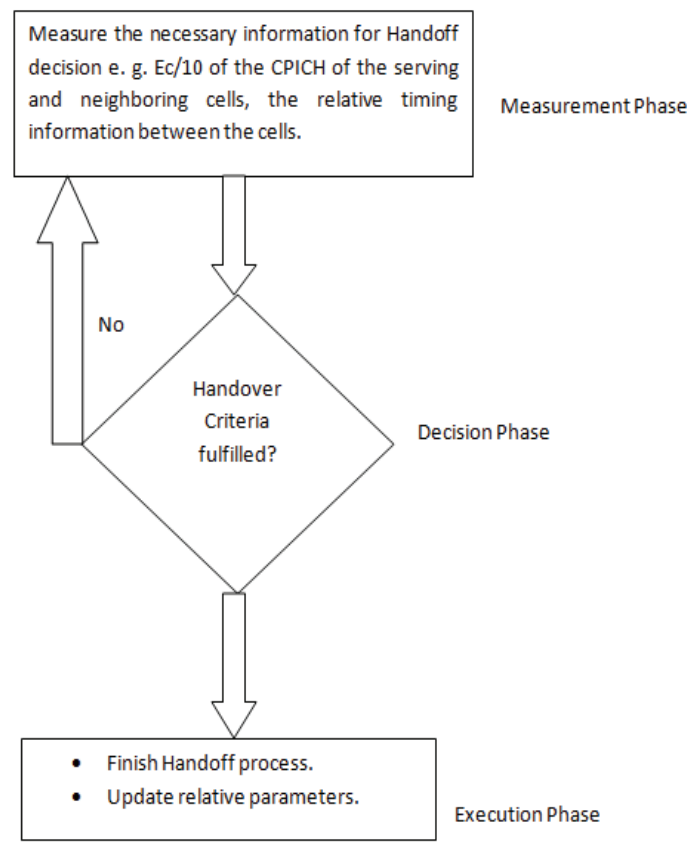

Figure 1. Different phases of handoff initiation.

In the CDMA system, soft handoff mechanism is used as discussed already. But it is necessary for the system to control the handoff initiation time. It means there should not be too early or too late handoff. Since too early or too late handoff affects the QoS of the system. Therefore to mitigate 
this problem, RSS (received signal strength) threshold has been used in this paper. RSS threshold provides the enough time to the mobile users to initiate the handoff process [5, $12,13]$.

It is also necessary to choose the threshold value very carefully in order to control the handoff time. By considering this factor, QoS has been improved and avoids too early and too late handoff initiation. This factor is called as adaptive RSS threshold (Thresmin). If any mobile user moves out of the serving cell's coverage region, then Thresmin helps in determining the handoff decision. This complete process is carried out with in very less time, due to which call dropping probability is decreased.

Quality of service (QoS) is measured in terms of call blocking probability and call dropping probability. The call is blocked if there are no available channels. The blocking probability can be obtained from the analysis of an $M / M / n / n$ queue. In general, the first $\mathrm{M}$ indicates call arrivals are modeled as Poison process with arrival rates of $\lambda$ call $/ \mathrm{s}$, the second $\mathrm{M}$ refers to exponential service time with mean $1 / \mu$ $\mathrm{s} / \mathrm{call}$, the first $\mathrm{n}$ refers to the number of channels, and finally, the second $\mathrm{n}$ refers to maximum number of acceptable users before blocking occurs. The famous Erlang-B equation (also called blocked calls cleared formula) [10] under the conditions of $\mathrm{M} / \mathrm{M} / \mathrm{n} / \mathrm{n}$ is given by:

$$
P_{B}=\frac{(\lambda / \mu)^{n} / n !}{\sum_{k=0}^{n}(\lambda / \mu) /}
$$

\section{Simulation Results and Performance MEASURE}

The simulations of the proposed algorithm are performed in MATLAB, following assumptions and parameters are taken for simulation, as tabulated in Table I.

TABLE I.

SIMULATION PARAMETERS

\begin{tabular}{|l|l|}
\hline \multicolumn{1}{|c|}{ Parameters } & \multicolumn{1}{c|}{ Values } \\
\hline Number of users & 70 \\
\hline Radius of cell & $1.5 \mathrm{~km}$ \\
\hline Neighboring cells & 8 \\
\hline Carrier Frequency & $500 \mathrm{MHz}$ to $1000 \mathrm{MHz}$ \\
\hline Mobile Station Height & $1 \mathrm{~m}$ to $10 \mathrm{~m}$ \\
\hline Capacity of serving cell & 1 to 8 \\
\hline Antenna Heights & $30 \mathrm{~m}$ to $200 \mathrm{~m}$ \\
\hline Radio propagation model & Hata Okumura path loss model and \\
free space path loss model
\end{tabular}

Probabilities of call blocking (CB) and handoff call dropping (HCD) are analyzed for urban area. Analysis is carried out by keeping the channels reserved with respect to relative mobility.

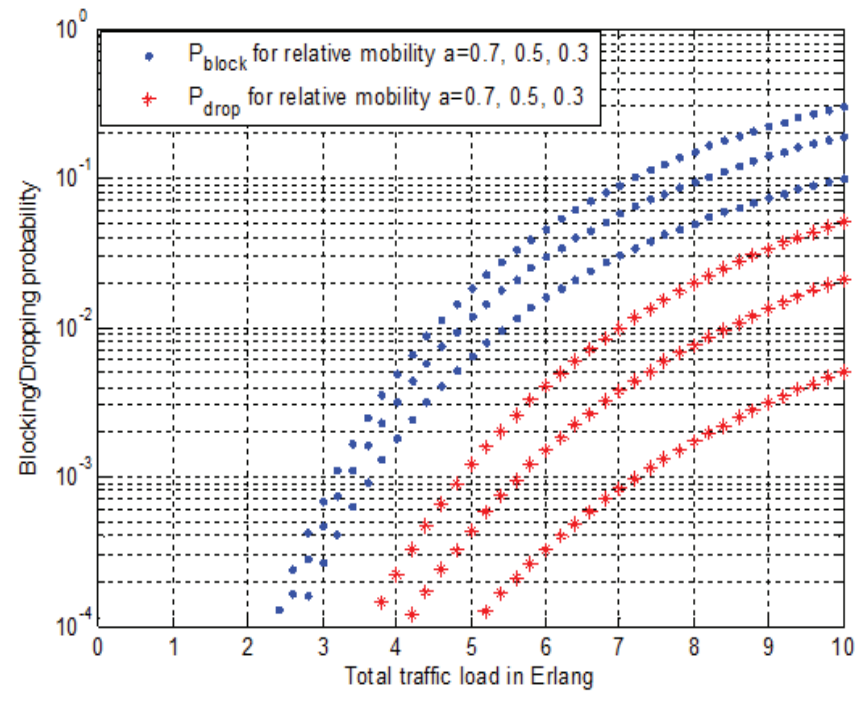

Figure 2. $\mathrm{CB}$ and $\mathrm{HCD}$ probabilities for different relative mobility (Reserved Channels $=3$ )

Call blocking (CB) probability is measured as the probability of call blocked when any user tries to attempt it. It can be easily realized that at a reasonable increase in traffic load, both the new blocking and handoff dropping probability will increase significantly. Also a decrease in relative mobility will decrease blocking and dropping probabilities of ongoing calls. It can also be observed as the number of reserved channels decreased, call blocking probability is also comparatively improved.

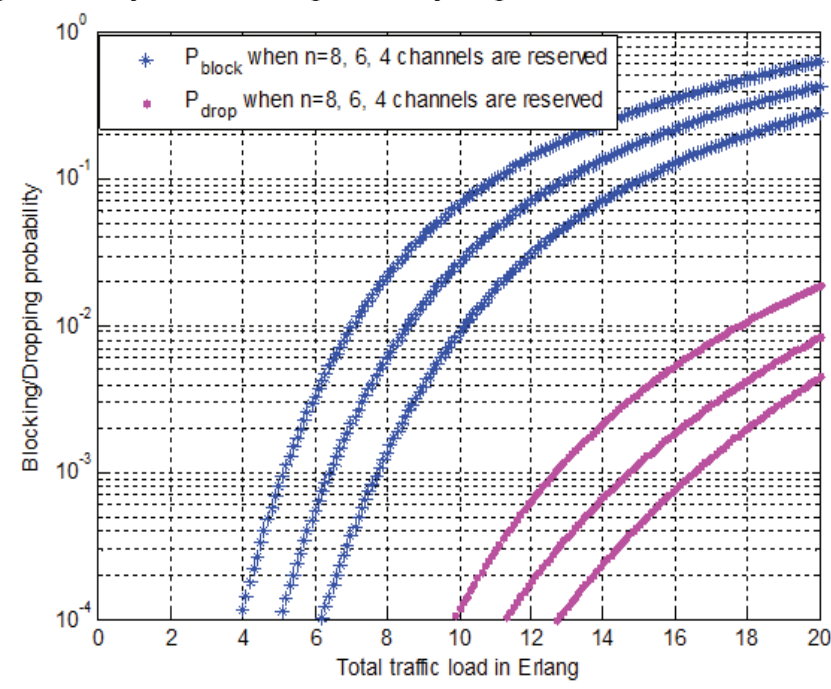

Figure 3. $\mathrm{CB}$ and $\mathrm{HCD}$ probabilities for different reserved channels for relative mobility $a=0.7$

Simulations have been carried out in MATLAB software with different values of relative mobility to measure the call blocking (CB) and handoff call dropping (HCD) probability. Call dropping probability is the measure of the probability when any ongoing call terminates. It is due to the reasons of 
handover failure. It is measured in the form of metric as handover blocking probability.

Traffic intensity across the entire radio system is known as traffic load. It is measured in Erlangs. It measures the number of handoff per call. If relative mobility increases, number of handoff per call also increases $[5,9]$.

In some cases, the results have been analyzed when some of the channels are kept reserved. Total traffic load is measured in Erlang, Relative mobility and total number of channels are represented by $a$ and $\eta$ respectively. Results obtained are shown in Fig. 2, Fig. 3, Fig. 4, and Fig. 5.

It can be observed that there is trade-off between call blocking (CB) and handoff call dropping (HCD). If service provider has the main concern for low handoff call dropping, then it is required to keep more channels reserved for it. If more channels are reserved for handoff call dropping, then the probability of blocking of new calls is increased.

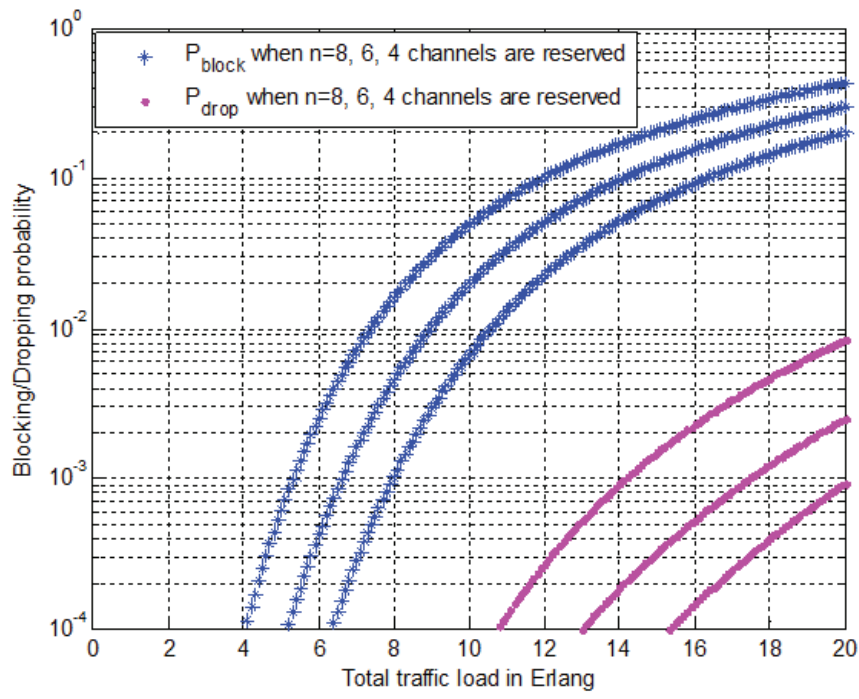

Figure 4. CB and HCD probabilities for different reserved channels for relative mobility $a=0.4$

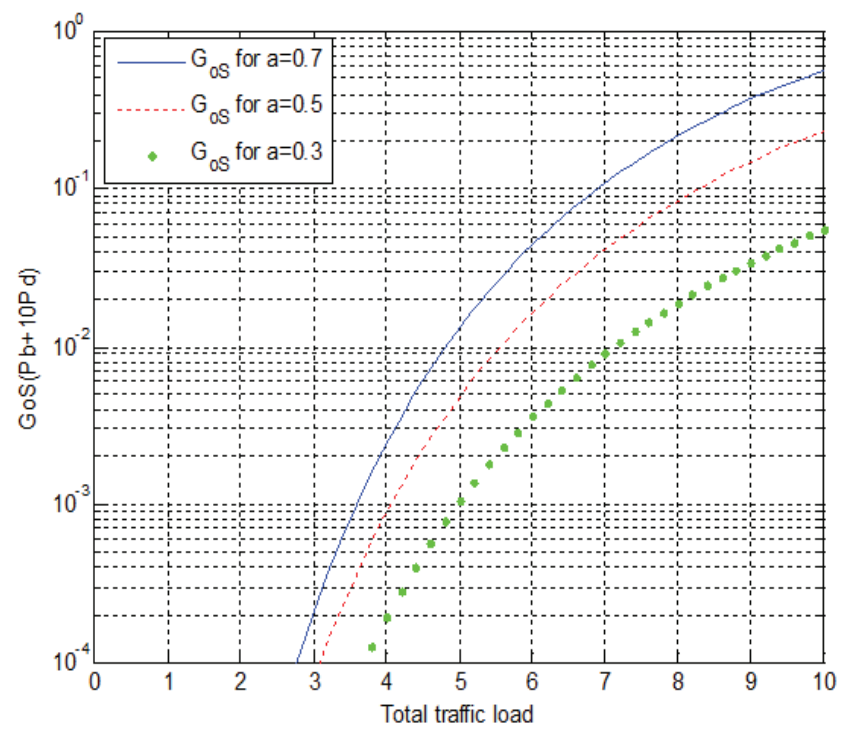

Figure 5. GoS for different relative mobility ( 3 channels are kept reserved)
In the dense population area, service provider divides the region into smaller cells to increase the capacity. As the number of cells and handoff are directly proportional to each other. A mobile user has to cross many boundaries of the cell for smaller cells and handoff occurred frequently. This type of scenario is happened in dense urban area. Therefore, this frequent handoff process degrades the QoS and increased the $\mathrm{CB}$ and HCD. The performance is measured by evaluating the blocking probability of the calls which is going to be start and dropping of the calls which are ongoing. Grade of Service (GoS) is a measure of congestion which is determined as the likelihood of a call being blocked, or the likelihood of a call being deferred past a specific measure of time [7], which is shown in Fig. 5.

TABLE II.

CALL BLOCKING CONDITIONS

\begin{tabular}{|c|c|c|c|c|}
\hline $\begin{array}{c}\text { Channel } \\
\text { Condition } \\
\text { Condition }\end{array}$ & 1 & 2 & 3 & 4 \\
\hline $\mathrm{G}$ & $\mathrm{SL}$ & $\mathrm{SL}$ & $\mathrm{SL}$ & $\mathrm{BL}$ \\
\hline $\mathrm{N}$ & $\mathrm{SL}$ & $\mathrm{SL}$ & $\mathrm{BL}$ & $\mathrm{BL}$ \\
\hline $\mathrm{H}$ & $\mathrm{SL}$ & $\mathrm{BL}$ & $\mathrm{SL}$ & $\mathrm{BL}$ \\
\hline $\mathrm{R}$ & $\mathrm{BL}$ & $\mathrm{BL}$ & $\mathrm{BL}$ & $\mathrm{BL}$ \\
\hline
\end{tabular}

Table II shows the possible cases of the blocked new calls attempt. The number of blocked new call attempts $(N B)$ can be read as follows:

\section{BL: Blocked, SL: Successful}

G: Mobile user receives the signals from both base station i.e. BS1 and BS2.

$\mathrm{N}$ : BS1's signal is acceptable but BS2 is not acceptable.

$\mathrm{H}$ : BS2's signal is acceptable but BS1 is not acceptable.

R: Signals from BS1 and BS2 are not acceptable.

1: Channel available at BS1 and BS2.

2: Channel available at BS1 but not available at BS2.

3: Channel available at BS1 but not available at BS2.

4: No channels are available either at BS1 or at BS2.

Therefore, the calls which are observed as blocked can be calculated as:

$$
\begin{aligned}
& N B=N M 4+N O 3+N O 4+N H 2+N H 4+N J 1+N J 2+ \\
& N J 3+N J 4
\end{aligned}
$$

The blocking probability $(P B)$ is defined by:

$$
P B=\frac{N B}{N G}
$$


Here, $N G$ represents the new calls attempts that are generated for establish new connection.

Table III shows all the possible cases in which the ongoing call is dropped due to too late initiation of handoff or improper handoff.

\section{DP: Drop, ND: Not dropped}

$\mathrm{K}$ : Ongoing serving link quality is acceptable.

$\mathrm{U}$ : Ongoing serving link quality is not acceptable or up to the mark, but alternative link quality is good.

T: Neither ongoing link quality nor alternative link's quality is acceptable.

1: Handoff process is initiated with availability of the channels in alternative BS.

2: Handoff process is initiated when there is no channel available in the neighboring cell's base station.

3: Handoff process is not started.

TABLE III.

HCD CONDITIONS

\begin{tabular}{|c|c|c|c|}
\hline Signal Condition & 1 & 2 & 3 \\
\hline $\mathrm{K}$ & $\mathrm{ND}$ & $\mathrm{ND}$ & $\mathrm{ND}$ \\
\hline $\mathrm{U}$ & $\mathrm{ND}$ & $\mathrm{DP}$ & $\mathrm{DP}$ \\
\hline $\mathrm{T}$ & $\mathrm{DP}$ & $\mathrm{DP}$ & $\mathrm{DP}$ \\
\hline
\end{tabular}

Therefore, the number of ongoing calls which are observed as dropped due to improper handoff can be calculated as:

$$
N D=N F 2+N F 3+N G 1+N G 2+N G 3
$$

The forced termination probability $(P F T)$ is defined by:

$$
P F T=\frac{N D}{(N G-N B)}
$$

The simulation observations show that $\mathrm{CB}$ and $\mathrm{HCD}$ probabilities are increased with increase in traffic load. As there is more traffic load, the more channels become busy. It can also be observed from the simulation results that $\mathrm{CB}$ and HCD probability are decreased with increase in relative mobility, even after keeping the traffic load constant. It shows that relative mobility also affects the system performance, as more mobility results in more handoff; due to which handoff mechanism is not performed well especially in the areas where the coverage is less. It can be seen that the constrained end likelihood abatements and watches out for a base level as the offered traffic diminishes. At the point when the offered traffic is low, the constrained terminations are fundamental because of handoff starts.

\section{CONCLUSIONS}

In this paper, variation in relative mobility with traffic load in wireless mobile network has been analyzed, which balances the traffic load according to the relative mobility in heterogeneous mobile network. It can be observed that there is trade-off between call blocking (CB) and handoff call dropping (HCD). If service provider is having the main concern for low handoff call dropping, then it is required to keep more channels reserved for it. If more channels are reserved for handoff call dropping, then the probability of blocking of new calls is increased. As a result, capacity of the serving cell is decreased. It can also be easily realized that GOS decreases with increase in traffic load, because with the increase of traffic load, more channels are required for keeping the call blocking and call dropping probability low.

\section{REFERENCES}

[1] A.E. Xhafa and O.K. Tonguz, "Handover performance of priority schemes in networks" IEEE Trans. Vehicular Tech., vol. 57, no. 1, pp. 565-577, Jan. 2008.

[2] G. Sharma and A. Kumar, "Fuzzy logic based 3D localization in wireless sensor networks using invasive weed and bacterial foraging optimization," Telecommunication Systems, vol. 67, no. 2, pp. 149162, May 2017.

[3] G. Sharma and A. Kumar, "Modified Energy-Efficient Range-Free Localization Using Teaching-LearningBased Optimization for Wireless Sensor Networks," IETE Journal of Research, vol. 64, no. 1, pp. 124-138, Jul. 2017.

[4] Kim, D., Sawhney, M. and Yoon, H., "An effective traffic management scheme using adaptive handovertime in next generation cellular networks" Int. Journal of Network Management, pp. 139-154, 2007.

[5] K. I. Itoh and S. Watanabe and J. S. Shih and T. Sato, "Performance of handoff algorithm based on distance and RSSI measurements," IEEE Trans. Vehicular Technology, vol. 51, no. 6, pp. 1460-1468, Nov. 2002.

[6] G. P. Pollini, "Trends in handover design," IEEE Comm. Magazine, vol. 34, pp. 82-90, March.1996.

[7] D. K. Panwar and L. Shyam, "Coverage analysis of hand off algorithm with adaptive hysteresis margin, "Proc.of 10th International Conference on Information Technology, pp. 133-138, Dec. 2007.

[8] S. H. Wie, J.-S. Jang, B.-C. Shin and D.-H. Cho, "Hand off analysis of the hierarchical cellular system," IEEE Trans. Vehicular Technology, vol. 49, no. 5, pp. 2027-2036, Sept. 2000.

[9] G. Sharma, \& A. Kumar, "Improved DV-Hop localization algorithm using teaching learning based optimization for wireless sensor networks". Telecommunication Systems, vol. 67, no. 2, pp. 163-178, 2017.

[10] M. Ruggieri, F. Graziosi and F. Santucci, "Modeling of the handover dwell time in cellular mobile communications Systems," IEEE Trans. Vehicular Technology, vol. 47, no.3, pp. 489-498, May. 1998

[11] G. Sharma, \& A. Kumar,"Dynamic Range Normal Bisector Localization Al gorithm for Wireless Sensor Networks". Wireless Personal Communications, vol. 9, no. 3, pp. 4529-4549, 2017. 
[12] R. Verdone and A. Zanella, "Performance of Received Power and Traffic-Driven Handover Algorithms in Urban Cellular networks", IEEE Wireless Communication, pp. 60-71, February 2002.

[13] Nandakumar S., Singh Rahul \& Singh Sanjeet, "Traffic Driven \& Received Signal Strength Adaptive Handoff Scheme," Int. Journal of Comp. App. (0975-8887), vol. 21, no.6, May 2011. 